\title{
Integration of low-cost supervisory mobile robots in domestic wireless sensor networks
}

\author{
Óscar Pascual García \\ Department of Systems Engineering \\ and Automation \\ Universidad Carlos III de Madrid \\ Leganés (Madrid), Spain \\ opascual@ing.uc3m.es
}

\author{
Alberto Brunete \\ Centre for Robotics and Automation \\ (CAR) \\ Universidad Politecnica de Madrid \\ Madrid, España \\ alberto.brunete@upm.es
}

\author{
Mohamed Abderrahim \\ Department of Systems Engineering \\ and Automation \\ Universidad Carlos III de Madrid \\ Leganés (Madrid), Spain \\ mohamed@ing.uc3m.es
}

\begin{abstract}
This paper describes how to introduce a supervisory low-cost mobile robot as part of a domestic Wireless Sensor Network (WSN), based on the ZigBee protocol. The robot is able to communicate with the other elements of the network using the same protocol of communication, and can be controlled remotely from a central PC. The paper outlines the architecture of the system and the configuration issues that have to be taken into account to set up the network.
\end{abstract}

Index Terms - WSN, mobile robot, ZigBee.

\section{INTRODUCTION}

The increasing momentum that home automation field and domestic robots are gaining in our society, and the big number of possible applications they have, demands the need to create systems where both, robots and home automation components, can work simultaneously and operate using the same communication protocol within the same network.

Current smart homes are moving from conventional remote-controlled houses to intelligent environments [1]. Today's smart homes are limited by the infrastructure they have been designed with: sensors and actuators. But in order to overcome this limitation, smart houses need to be able to "move", to be free to perform tasks. This can only happen if the smart house counts on a robot to perform these new tasks.

Smart houses need to interact with the users as people do, and this can only be possible with robots that can perform the same actions as human do and behave as humans do. This paper presents a new system to integrate robots and wireless devices from different vendors. The main objectives addressed in this paper are to implement a Wireless Sensor Network based on ZigBee protocol, to show all the WSN information on the PC screen, to control the robot remotely, to communicate all the different elements of the network and to prove the compatibility between different platforms and different operation modes.

The solution presented in this paper has two main parts: a Wireless Sensor Network (WSN) based on ZigBee protocol, and a mobile robot. The whole system is controlled and supervised with a computer.

WSN contains two sensor/actuator nodes, and one USBGateway connected to a PC. It is based on ZigBee protocol, where the Gateway is the coordinator. The sensor/actuator nodes take care of physical conditions monitoring, and transmit this information to the Gateway. The USB-Gateway receives the ZigBee information from the sensor nodes and transmits it to the PC. It is also used to control the mobile robot remotely.

The mobile robot receives control commands from PC and executes them. The communication with the computer can be bidirectional, since the robot is also able to work as another sensor node, measuring temperature or communicating its location. Moreover, the robot is able to interact with the other components of the network (sensor/actuator nodes) using the same protocol of communication. The prototype robot is made using low-cost 3D printing technology and low-cost electronics (Arduino based).

The structure of the paper is as follows. Section II presents related state of the art. Section III describes the architecture of the system. Section IV shows the communication system and section $\mathrm{V}$ presents the control interface.

\section{STATE OF THE ART}

\section{A. Wireless Sensor Network (WSN)}

A WSN is a group of distributed autonomous sensors in charge of monitoring physical and environment conditions. Those elements, called "motes" or "nodes", are able to communicate between each other and with a Gateway that receives all the information from the sensors. Therefor, a WSN is formed by: sensor/actuator nodes and a gateway. Additionally, routers can be added to extend the range and the routing capability of the network.

The origin of these networks is related to military applications, started with the DSN (Distributed Sensor Networks) project developed by DARPA (Defense Advanced Research Projects Agency).

The concept "mote" comes from the Smart Dust research proposal written by Kristofer Pister, et al. [2]. The aim of the project was autonomous sensing and communication in a cubic millimeter.

The principal characteristics of these networks are the low energy consumption, the low cost and the small 
dimensions of the sensor nodes. Nowadays they are used in many different applications like industrial sensing, high security environments, energy harvesting, medicine and home automation, among others.

To communicate the information of the sensor nodes, several wireless communication technologies can be used, such as WiFi, Bluetooth, Z-Wave, EnOcean, or ZigBee. In this case ZigBee is the one selected, because this protocol is one of the most used in this kind of WSNs and it is the principal alternative to conventional wired home automation networks. ZigBee also allows the creation of flexible systems, where new elements can be added easily without changing the configuration of the whole network, and the most important characteristic is its low energy consumption. In addition, the ZigBee Alliance provides different standards which give product manufacturers a straightforward way to help their customers gain greater control of, and even improve, everyday activities. They take full advantage of ZigBee's many strengths so products using ZigBee low-power wireless standards can be easily installed and allowed to run on harvested energy or batteries for years. This simplicity makes them easy to use and gives consumers and businesses the tools they need to have greener homes and offices. Some of this standards or profiles are: ZigBee Building Automation, ZigBee Home Automation, ZigBee Smart Energy, or ZigBee Remote Control.

\section{B. Supervisory Mobile Robots}

Robots integrated in the WSN can receive information directly from the network and act consequently. They can act as mobile sensors: they can feel the same as humans do, while standard sensors (i.e. illumination, temperature, noise) are placed in the wall or floor, (providing localized readings, which are inaccurate measurements).

Most of supervisory mobile robots are limited to exploration with a camera or its own sensors [3][4][5]. They hardly communicate with the devices in their environment directly. Among the ones that do communicate directly are CompanionAble and NurseBot [6][7].

CompanionAble [6] provides the synergy of Robotics and Ambient Intelligence technologies and their semantic integration to provide for a caregiver's assistive environment. This supports the cognitive stimulation and therapy management of the care-recipient. This is done by a robotic companion that works collaboratively with a smart home environment (stationary facilitation).

The CMU Nursebot [7], Pearl, was designed to interact with elders in assisted living facilities and nursing homes. The robot was deployed in field tests in the Longwood Retirement Community in Oakmont, PA. It reminds elders of planned activities on their calendars and would lead them to the activity if necessary. The research conducted on the Nursebot focus on autonomous navigation, activity planning, cognitive prosthetics, and human-robot interfaces. However, Nursebot is not able to interact with the environment manually and so it could not effect work on behalf of the user.

Furthermore, DHCompliant [8] is an example of integration of smart homes and robots. DH Compliant is a platform that provides a framework to interconnect and communicates digital homes and robot devices. It is also a protocol gateway for the most common home automation protocols.

\section{OVERALL SYSTEM DESCRIPTION}

The proposed system is formed by a Wireless Sensor Network simulating a domestic network, and a mobile robot. The sensor nodes of the WSN take information of the physical and environment conditions (temperature, luminosity, presence, etc.) and send this parameters to the Gateway using the ZigBee protocol. The Gateway is connected to a central PC (running GNU/Linux Ubuntu 12.04), and it transmits the information through a USB port, so the information can be treated and displayed in the PC.

At the same time the $\mathrm{PC}$ is receiving the sensor parameters, the mobile robot can be controlled from this PC. For this purpose, a serial monitor can be used, and all written commands will be transmitted via ZigBee through the Gateway to the robot. It can also be controlled using a graphic interface made with Processing, where the control of the robot is much easier to perform. In addition, the robot can work as another sensor node and it can send information back to the PC (temperature and position). The whole system schematic is shown in Fig.1.

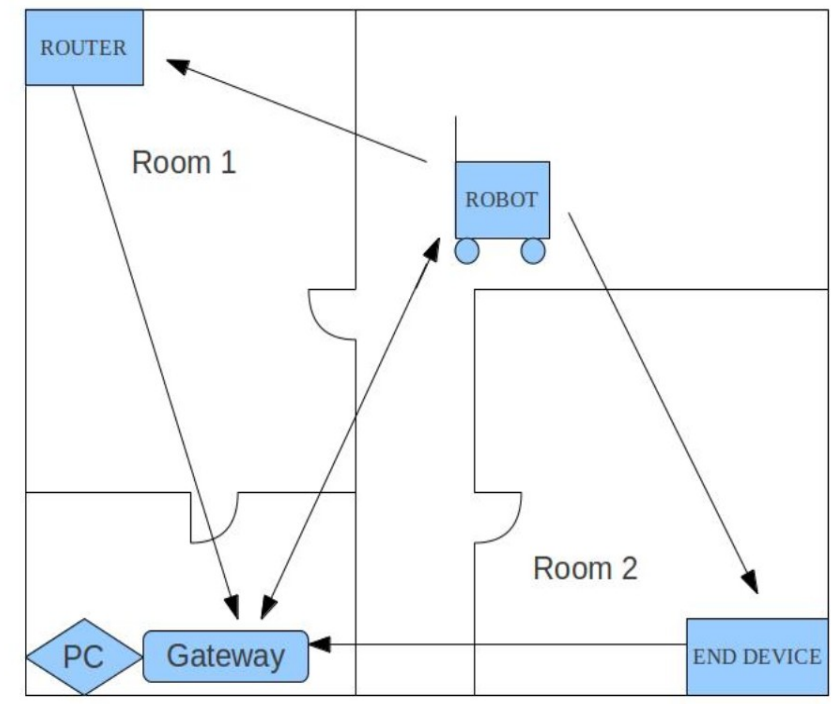

Fig. 1 System description layout.

\subsection{WSN}

The implemented domestic wireless network is formed by two sensor/actuator nodes and one USB-Gateway. The components selected belong to Libelium Company. This company provides a node (Waspmote) with low energy consumption, small size and the possibility to add a big 
number of different sensors, thanks to their expansion sensor boards.

\section{A. Waspmotes}

Two Waspmotes are used in the WSN, where one is configured as a Router and the other as an End Device, so in the same network all the possible configurations for a ZigBee component (Coordinator, Router and End Device) find place. In Fig.2. one of the Waspmotes used and one Waspmote Event Sensor Board are shown. There are many different Expansion Sensor Boards for Waspmotes (such as Gases, Events, Smart Cities or Agriculture boards) and many different kind of sensors so, depending on the application one board is more suitable than the others.
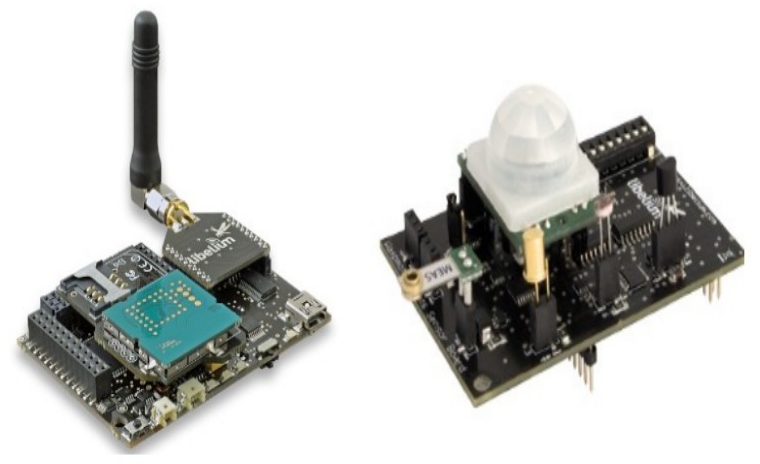

Fig. 2 Libelium Waspmote and Event Sensor Board.

\section{B. USB-Gateway}

The Gateway connected to the computer is configured as Coordinator, which is the device that sets up the network. It also receives all the information sent from the Waspmotes, transmits it to the $\mathrm{PC}$, and sends the control commands to the robot. The Gateway module is shown in Fig. 3.

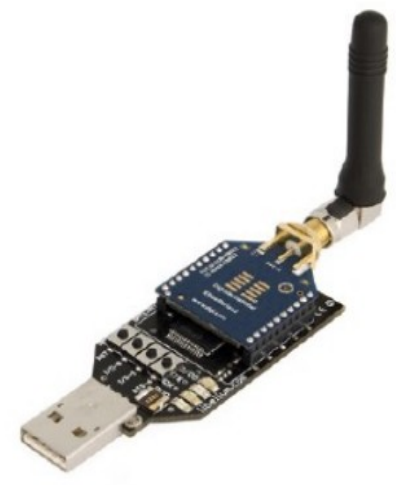

Fig. 3 Libelium USB-Gateway.

\subsection{MOBILE ROBOT}

The structure of the robot is designed with OpenSCAD, an open source CAD program, and then it is developed using 3D printing technology.

\section{A. Hardware}

To control the robot, an Arduino Due micro-controller board is used. This low cost open source platform has a sufficient number of input/output for our application. In addition to this Arduino board, an Xbee Shield is necessary to implement the wireless communication,

For the power supply, a 7.4V Lipo Battery (2200mAh) has been chosen. This battery gives the robot quite a good autonomy.

Finally, in order to move the wheels, two Hitec HS-311 Standard servos are used.

\section{B. Software}

Since the board used to control the robot is an Arduino, and this platform has its own IDE (Arduino IDE), the programming of the micro-controller is performed using this software. Besides, Arduino has its own libraries to control the movement of the servos.

The prototype mobile robot is shown in Fig.4.

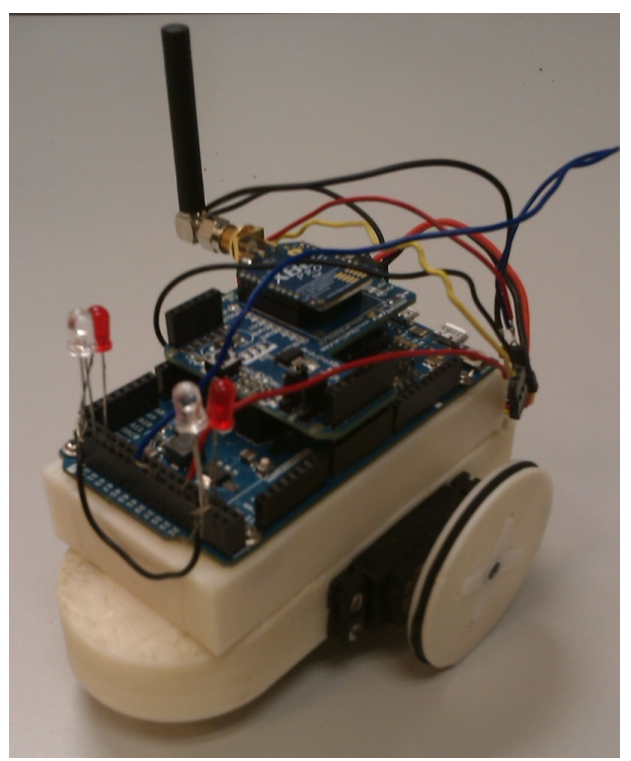

Fig. 4 Mobile Robot (Arduino based).

\section{COMMUNICATION SYSTEM}

The system is composed of four different modules of communication with different configurations, and two different hardware platforms.

The first step, is to configure the communication modules (Xbee ZigBee modules). These Xbee modules belong to Digi International ${ }^{\circledR}$ Inc. To configure the modules, X-CTU 
software is used. When configuring them, two different serial interfaces can be selected, Transparent Operation or API Operation. This is an important step because depending on the characteristics of the network, one operation mode is more suitable than the other, or even only one of them works.

At this point, the main problem of this work appeared, since the Libelium gateway is designed to receive data but not to send it, and there is very few information about this issue. To solve this problem, the module of communication plugged into the gateway needs to be configured in AT Operation Mode (Transparent Operation) so that all written commands on the computer keyboard are transmitted via ZigBee to the destination point. With this configuration the gateway is able to receive data but also to send data.

A) Transparent Operation (AT): when operating in transparent mode, the modules act as a serial line replacement. All UART data received is queued up for RF transmission.

B) API (Application Programming Interface): API operation is an alternative to transparent operation. The frame-based API extends the level to which a host application can interact with the networking capabilities of the module. When in API mode, all data entering and leaving the module is contained in frames that define operations or events within the module.

Once the operation mode is selected, the second step is to select the configuration of the mote in the ZigBee network. There are three different possibilities: Coordinator, Router or End Device. Tab.1.

The communication modules are configured as shown in

\begin{tabular}{|c|c|c|}
\hline $\begin{array}{c}\text { ZigBee } \\
\text { Configuration }\end{array}$ & Operation Mode & Hardware \\
\hline Coordinator & AT & Gateway \\
\hline End Device & API & Waspmote 1 \\
\hline Router & API & Waspmote 2 \\
\hline End Device & AT & Arduino \\
\hline
\end{tabular}

Tab.1. Configuration of the communication modules.

The Coordinator and the Robot are configured in AT operation mode to have a fast and robust communication. All the commands written in the keyboard of the PC are sent through the Gateway and received in the Robot. If the Coordinator is configured in API mode, every time a command is written on the keyboard, in order to send it, a whole frame must be built and sent, so the communication is more complex and slower. This operation mode is useful when the network has a lot of nodes and there are a big number of messages flying around. The structure of an API frame is shown in Fig. 5.

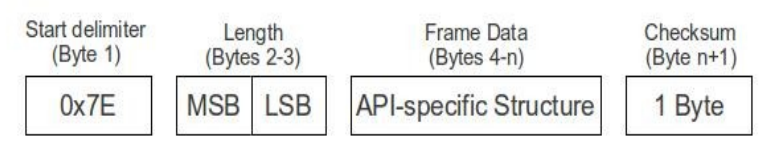

Fig. 5 API frame structure.

The Router and the End Device are configured in AT mode to prove that both configurations are compatible and elements with different operation mode can work in the same network and they can communicate between each other.

The final step is to configure the specific parameters of the communication protocol such as PAN ID, Destination Address High, Destination Address Low and Baud Rate, To do it, the X-CTU software is also used.

Devices parameters are specified in Tab.2.

\begin{tabular}{|c|c|c|c|c|}
\hline & $\begin{array}{c}\text { Waspmote 1 } \\
\text { (End Device) }\end{array}$ & $\begin{array}{c}\text { Waspmote 2 } \\
\text { (Router) }\end{array}$ & $\begin{array}{c}\text { Coordinator } \\
\text { (Gateway) }\end{array}$ & $\begin{array}{c}\text { Mobile } \\
\text { Robot } \\
\text { (Arduino) }\end{array}$ \\
\hline PAN ID & 222 & 222 & 222 & 222 \\
\hline $\begin{array}{c}\text { Destination } \\
\text { Address } \\
\text { High }\end{array}$ & 0013A200 & 0013A200 & 0013A200 & 0000 \\
\hline $\begin{array}{c}\text { Destination } \\
\text { Address } \\
\text { Low }\end{array}$ & 408 D638B & 408D638B & 408D50BB & FFFF \\
\hline Baud Rate & 38400 & 38400 & 38400 & 38400 \\
\hline
\end{tabular}

Tab.2. Configuration of the elements.

All the components need to have the same PAN ID (222), and all must be configured with the same Baud Rate (38400). The destination addresses are configured so they can communicate as shown in Fig. 6. 


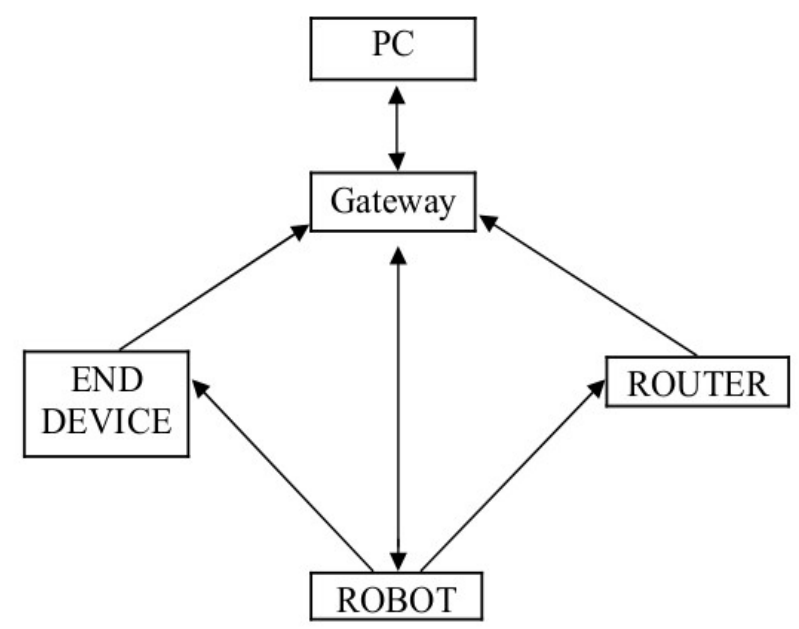

Fig. 6 Communication diagram.

Both sensor nodes (End Device and Router) only send messages to the Gateway (which Source Address Low is 408D638B), and the Gateway only sends messages to the Robot (which Source Address Low is 408D50BB), but the Robot must send messages to all the components of the network, so it has to be configured in Broadcast Mode (Destination Address High $=0000$, and Destination Address Low $=$ FFFF $)$.

Another important fact to have in consideration is the compatibility between two different hardware platforms, these platforms are Libelium Waspmotes and Arduino Due. To communicate the Arduino board with the rest of the elements, the only thing to do is to include the Arduino Zigbee API library. With this library, it is easy to send and receive Zigbee messages.

\section{CONTROL INTERFACE}

A graphic interface is developed in order to improve the control of the robot. Thanks to this interface, it is very easy to control the movement of the mobile robot, or to activate its other functions, just with a mouse click. The final version of the developed interface is shown in Fig. 7.

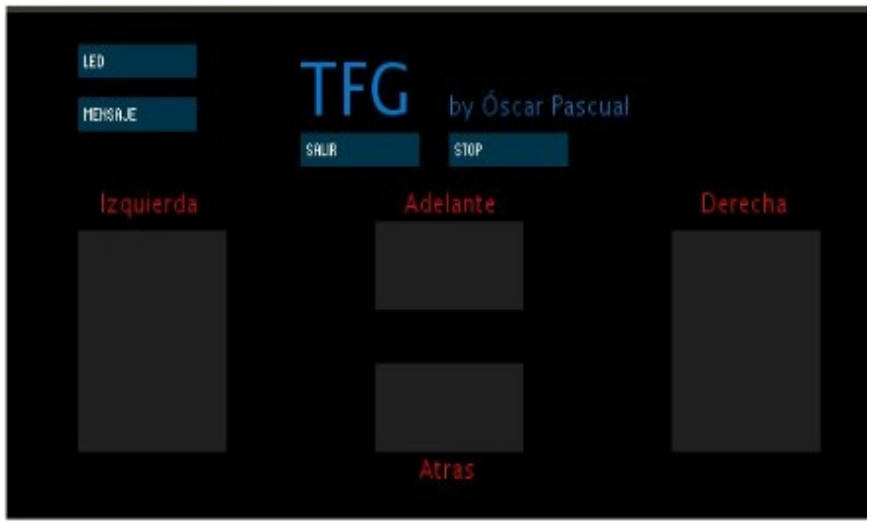

Fig. 7 Processing control interface.

This interface has been developed using Processing. Processing is an open source IDE and also a programming language based on java.

In the interface, there are a few control commands to click on to turn right/left, go forward and backward, stop, light the LEDs on/off, send a message to the gateway, (i.e. position and temperature), and exit and close the interface.

When any of these functions is pressed, the processing application send the corresponding character to the port where the Gateway is connected. This command is received in the DIN pin of the Gateway (Data Input pin) and queued for transmission. When the transmission is possible, the command goes through the DOUT pin (Data Output pin) and it is transmitted via ZigBee to the appropriate destination. So, the interface sends a specific command to the Gateway and this one actuates as a wire replacement.

\section{CONCLUSIONS AND FUTURE WORK}

This study opens an important number of possibilities and future research lines, because the home automation field and the wireless sensor networks have a considerable number of applications and in the next few years they will be deployed in many homes and industries. The inclusion of a mobile robot with the ability to interact autonomously with the components installed in any home, will certainly improve considerably the security, comfort, and the life quality of people, especially in the case of old people and those with some kind of impairment. This robot can take care of many useful tasks such as physical and environment conditions monitoring, checking the correct working of all systems, activate actuators and communicate emergencies among many others.

Some possible future research lines or improvements for this project are: giving the robot some intelligence, to be able to move or actuate autonomously, adding actuators to the network (light control, shutters, etc.), improving the control interface, developing an IP control system, integrating the robot in a wired home automation network (such as KNX) and creating a group or a team of supervisory robots working in collaboration. 


\section{REFERENCES}

[1] Balaguer, C. (2007). Towards robotic smart homes. In 24th International Symposium on Automation and Robotics in Construction.

[2] K. S. J. Pister, J. M. Kahn and B. E. Boser, "Smart Dust: Wireless Networks of Millimeter-Scale Sensor Nodes", Highlight Article in 1999 Electronics Research Laboratory Research Summary.

[3] Pinta, J. D. L., Maestre, J., Camacho, E., and Alonso, I. G. (2011). Robots in the smart home: a project towards interoperability. International Journal of Ad Hoc and Ubiquitous Computing, 7:192 - 201.

[4] Badii, A., Etxeberria, I., Huijnen, C., Maseda, M., Dittenberger, S., Hochgatterer, A., Thiemert, D., and Rigaud, A.-S. (2009). Companionable- mobile robot companion and smart home system for people with mild cog-nitive impairment. Journal of Nutrition, Health and Aging, 13:113.

[5] Pollack, M. E., Engberg, S., Matthews, J., Thrun, S., Brown, L., Colbry, D., Orosz, C., Peintner, B., Ramakrishnan, S., Dunbar-Jacob, J., McCarthy, C., Montemerlo, M., Pineau, J., \& Roy, N. (2002). Pearl: A mobile robotic assistant for the elderly. In AAAI workshop on automation as eldercare.

[6] Xiaofeng Xiong; Zhangjun Song; Jianwei Zhang, "Domestic robots with multi-function and safe internet connectivity," Information and Automation, 2009. ICIA '09. International Conference on, vol., no., pp.277,282, 22-24 June 2009 doi: 10.1109/ICINFA.2009.5204935

[7] Cakmak, M.; Takayama, L., "Towards a comprehensive chore list for domestic robots," Human-Robot Interaction (HRI), 2013 8th ACM/IEEE International Conference on, vol., no., pp.93,94, 3-6 March 2013 doi: 10.1109/HRI.2013.6483517
[8] Jayasiri, A.; Mann, G. K I; Gosine, R.G., "Mobile robot behavior coordination using supervisory control of Fuzzy Discrete Event Systems," Intelligent Robots and Systems, 2009. IROS 2009. IEEE/RSJ International Conference on , vol., no., pp.690,695, 10-15 Oct. 2009 doi: 10.1109/IROS.2009.5354780

[9] Baronti, P., Pillai, P., Chook, V.W., Chessa, S., Gotta, A., Fu, Y.F., 2007. Wireless Sensor Networks: a survey on the state of the art and the 802.15.4 and ZigBee standards.

[10] ZigBee Alliance Web site. [On-line]. (July 2013). Available on the Web: $<\mathrm{http}: / /$ www.zigbee.org/>

[11] Introduction to 3D Printing Technology. [On-line]. (July 2013). Available on the Web: <http://www.ptonline.com/articles/3d-printers-lead-growth-ofrapid-prototyping $>$

[12] OpenSCAD User Manual. [On-line]. (January 2013). Available on the Web: <http://en.wikibooks.org/wiki/OpenSCAD_User_Manual>

[13] Arduino Web site. [On-line]. (July 2013). Available on the Web: $<$ http://www.arduino.cc/ $>$

[14] Libelium Web site. [On-line]. (July 2013). Available on the Web: $<$ http://www.libelium.com/>

[15] Processing Web site. [On-line]. (August 2013). Available on the Web: $<$ http://processing.org/> 